the standard anesthetic choice despite being associated with postoperative complications. Regional anesthesia (RA) offers an alternative anesthetic choice. This study aimed to qualitatively review the literature on efficacy of RA alone for breast surgery.

Methods OVID and EMBASE databases were searched for articles pertaining to the use of regional anaesthesia alone for breast surgery up to June 2021. Systematic review was conducted according to PRISMA guidelines.

Results 24 studies were reviewed encompassing various RA blocks (paravertebral (PVB), pectoralis (PECS), thoracic and cervical epidural blocks (TEA, CE)). PVB represented $42 \%$ of studies. Mean patient age was from 54.8 - 72.6, most (65\%) were ASA class 2-3. Two studies found the RA group had significantly less pain $24 \mathrm{~h}$ after surgery, compared to GA. ${ }^{1,2}$ Similarly, opioid and morphine equivalent requirements were significantly higher for GA compared to RA in two studies., One study reported lower prevalence of pain in the TEA group versus GA. 3 Patients in the RA group had significantly less postoperative nausea/vomiting versus their GA counterparts, and greater level of patient satisfaction in two studies. ${ }^{2,4}$ Rate of conversion to GA was 0\% (95\% CI [0\%-3\%]). No significant difference between length of stay or days till resumption of diet was found.

Conclusions There is substantial data to support the effectiveness of PVB as an alternative to GA for breast surgery. Such data was limited for PECS blocks, further studies evaluating efficacy of these blocks is warranted.

\section{CLAVIPECTORAL FASCIAL PLANE BLOCK AND SUPRACLAVICULAR NERVE BLOCK FOR REMOVAL OF OSTEOSYNTHESIS MATERIAL FROM A CLAVICLE FRACTURE. WHY NOT?}

D Gonçalves, F Teixeira*, C Sousa, R Graça, P Miguelez, C Sampaio. Centro Hospitalar Trásos-Montes e Alto Douro, Vila Real, Portugal

\subsection{6/rapm-2021-ESRA.186}

Background and Aims Clavipectoral Fascial Plane Block (CPB) is most commonly used as an anesthesia and postoperative analgesia technique to clavicle fracture surgery. It consists in a local anesthetic injection under the clavipectoral fascia. The Supraclavicular Nerve from the Superficial Cervical Plexus is responsible for the sensory innervation of the skin that covers the clavicle, shoulder and superior region of the chest, It's blockage should complement the CPB.

Methods A 25-year-old male patient, ASA I, (weight $70 \mathrm{Kg}$, height $184 \mathrm{~cm}$ ) who underwent right clavicle fracture surgery under general anesthesia, was now scheduled for osteosynthesis material removal.

After obtaining informed consent to perform surgery under regional anesthesia, we performed an ultrasound-guided supraclavicular nerve block (SCB) and CPB with a total of $25 \mathrm{~mL}$ of local anesthetic $(12.5 \mathrm{~mL}$ Ropivacaine $0,75 \%$ and $12.5 \mathrm{~mL}$ of Mepivacaine 2\%), 5 and $20 \mathrm{ml}$ were administered respectively. The $\mathrm{CPB}$ was performed with two needle punctures one at each side of the osteosynthesis material.

Results A satisfactory peripheral nerve block was achieved, and no complications were found. The patient remained calm and hemodynamically stable throughout the entire procedure.

Conclusions The combination of the CPB with the SCB is a safe and easy to perform procedure. It allows risk reduction of phrenic nerve block as well as prevention of upper limb paralysis, when compared to brachial plexus block at the interscalene level. Other benefits are general anesthesia adverse effects avoidance, such as nausea and vomiting, sore throat, as well as airway manipulation.

\section{FEMORAL BRANCH BLOCK OF GENITOFEMORAL NERVE FOR FEMORAL ENDARTERECTOMY: A CASE REPORT}

${ }^{1} \mathrm{D}$ Maliamanis ${ }^{*},{ }^{1} \mathrm{C}$ Christofis, ${ }^{2} \mathrm{~A}$ Koureas, ${ }^{2} \mathrm{~S}$ Michaelides, ${ }^{2} \mathrm{~K}$ Kapoulas, ${ }^{1} \mathrm{C}$ Yiapana. ${ }^{1}$ General Hospital of Nicosia. Cardiac, Thoracic and Vascular Anaesthesia Department, Strovolos, Cyprus; ${ }^{2}$ General Hospital of Nicosia. Vascular Surgery Department, Strovolos, Cyprus

\subsection{6/rapm-2021-ESRA. 187}

Background and Aims Patients undergoing lower extremity vascular surgery are often at high risk for perioperative complications. We report a case of a high risk patient who underwent common femoral endarterectomy anesthetized by ultrasound guided femoral branch block of genitofemoral nerve (FBG) plus intravenous sedation.

Methods A 64 years old female patient was scheduled for common femoral endarterectomy and patch angioplasty. She had a history of congestive heart failure after myocardial infarction and chronic obstructive pulmonary disease. She was on dialysis three times a week, had echinococcal cyst in left lung, suffering from diabetic foot and was admitted to the hospital multiple times due to deregulated CHF. She was considered as high risk patient for general anesthesia. Her international normalized ratio (INR) was 1,7 so neuraxial anesthesia was contraindicated.

The FBG nerve provides sensation to the skin of the femoral triangle and to the underlying tissues and vessels within the femoral sheath. FBG nerve block was performed as a single peripheral nerve block for surgical anesthesia plus sedation. Using Mindray M7 ultrasound machine and L14-6Ns Linear transducer, the needle was inserted in plane to the sheath and $15 \mathrm{ml}$ of $0,5 \%$ ropivacaine was injected, as shown.

Results Adequate surgical anesthesia was achieved $30 \mathrm{~min}$ later. Extra local anesthesia was needed for the incision close

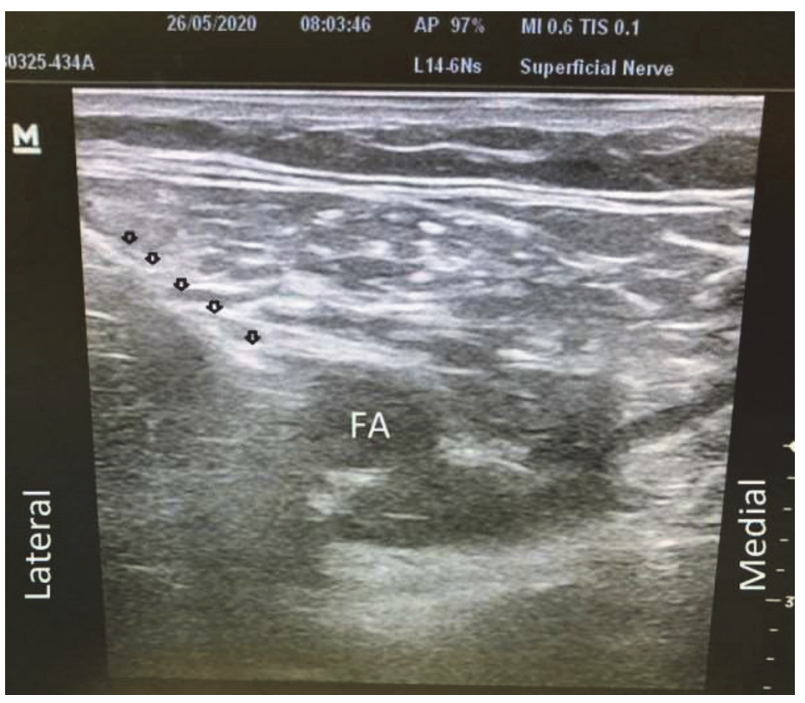

Abstract 187 Figure 1 